\title{
Power over fiber in Radio over Fiber Systems in 5G scenarios
}

\author{
C. Vázquez, Senior Member, IEEE, J.D. López-Cardona, D. S. Montero, I. Pérez, P. C. Lallana, and \\ Fahad M. A. Al-Zubaidi \\ Universidad Carlos III de Madrid, Avda de la Universidad 30, 28911, Leganés, Madrid, Spain \\ Tel: (34)916249191,e-mail address: cvazquez@ing.uc3m.es
}

\section{ABSTRACT}

5G verticals needs of dedicated power architectures for providing efficient systems. Some schematics and measurements on optical power delivery to remote nodes in 5G scenarios are described. Different types of optical fibers including plastic and silica optical fibers are considered.

Keywords: power over fiber, nonlinear effects, remote radio heads, optical fibers, multicore fibers, 5G.

\section{INTRODUCTION}

The mobile traffic explosion fosters 5G (5th Generation) cellular network evolution to increase the system rate around 1000 times higher than the current systems and to manage the expected monthly global mobile data traffic that was expected to surpass 24.3 exabytes this year [1]. Different verticals will be benefited from $5 \mathrm{G}$ deployment such as Vehicle to Everything (V2X) or Internet of Things (IoT) applications. V2X is a key technology enabler that enhances Advanced Driver Assistance Systems (ADAS), bringing significant value to a wide range of safety features. Power consumption demands of future 5G-based Remote Radio Heads (RRHs) are foreseen to be dramatically reduced, especially if Analog Radio over Fiber (ARoF) mobile fronthaul is considered [2]. Multiple RRHs should be deployed with less power consumption requirements to cope with Cloud/Centralized-Radio Access Networks, where processing is done at the Central Office (CO). Those new scenarios have opened up new application niches for Power over Fiber (PoF) technology [3]. A 100 GHz UniTraveling Carrier Photodiode (UTC-PD), with a low-power-consumption RF amplifier was proposed as part of a future 5G RRH. It was optically powered through a multicore fiber (MCF) with electrical powers of 80mW [4].

Hence, in this work we address the use of different types of optical fibers in PoF technology in future 5G scenarios. We compare theoretically the power levels that can be delivered and show some prototypes and experimental results of tenths and hundredths of $\mathrm{mW}$. The data transmission quality impact with simultaneous optical power delivery signals in both dedicated- and shared-core/fiber scenarios is also discussed.

\section{POWER OVER FIBER BASICS}

A schematic of a general PoF system is shown in Fig. 1 with a power delivery channel and an optional communication and control channel.

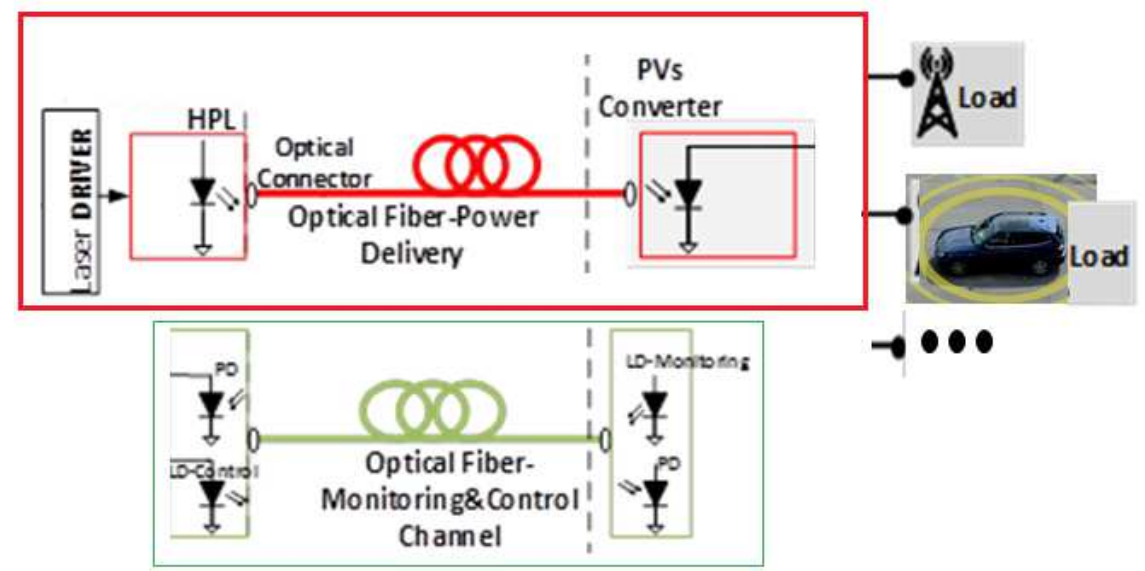

Figure 1: Schematic of PoF system with power and control (optional) channels. $5 G$ verticals loads.

The power channel includes a High Power Laser (HPL), the distributing optical fiber and the Photovoltaic (PV) converters, electronics to drive and control the laser, to optimize PV operation, and to adapt to the required voltage levels on the load. Different loads are considered depending on the $5 \mathrm{G}$ vertical to be supported.

In each scenario that requires a high bandwidth connection, optical fibers are used as the fronthaul or infrastructure to transmit those high bit rates. The type of optical fiber depends on the specific application, plastic optical fibers (POFs) are already installed in cars meanwhile, single mode optical fibers (SMFs) dominate in passive optical networks and some in building infrastructure includes multimode fibers (MMFs). Some 
experiments and specific designs aspects are considered for each fiber type in the next sections. But, in any case the overall efficiency of the PoF system (GEE) is given by:

$$
\text { GEE }=\frac{\text { Energy provided to Load at RAM }}{\text { Energy provided to HFL }}=N 1 \times N 2 x N 3
$$

where N1 is the electrical-to-optical conversion efficiency at the transmitter, N2 is the optical fiber transmission efficiency, N3 is the optical-to-electrical conversion efficiency of the PV converter at the remote node or load.

\section{MULTIMODE OPTICAL FIBER SCENARIOS}

We can consider either POFs or glass fibers (GOFs). POFs offer easy and cost-efficient connection, high safety, and extremely high flexibility, being currently installed mostly in cars, home and automation networks. As an example, using PoF technology in a car instead of copper cables in some ADAS parts, provides advantages such as weight reduction and immunity to electromagnetic interference.

\subsection{Specific characteristics}

The maximum amount of power that can be delivered to the remote site depends on the transmission loss, and the optical fiber damage threshold, $P_{t h}$. This damage threshold power, $P_{t h}$, is given by:

$$
P_{t h}=I_{\text {th }} \cdot A_{\text {eff }}
$$

where $A_{\text {eff }}$ is the fiber effective mode area that depends on the mode field diameter (MFD). As a first approximation, we estimate the MFD and an effective diameter, $\mathrm{d}_{\mathrm{eff}}$, as in [5]; being the effective area given by:

$$
A_{e f f}=\pi \cdot\left(\frac{d_{t f f}}{2}\right)^{2}
$$

The PoF systems based on MMFs can support higher powers than those based on SMFs; as MMFs have higher effective areas. The fiber damage power density threshold, $I_{t h}$ depends on the material. In silica fiber, $I_{t h}$ is around $2.5 \mathrm{MW} / \mathrm{cm}^{2}$ meanwhile in POFs is $6.6 \mathrm{~kW} / \mathrm{cm}^{2}[6]$. On the other hand, Fig. 2 shows the fiber attenuation influence on optical power delivery.
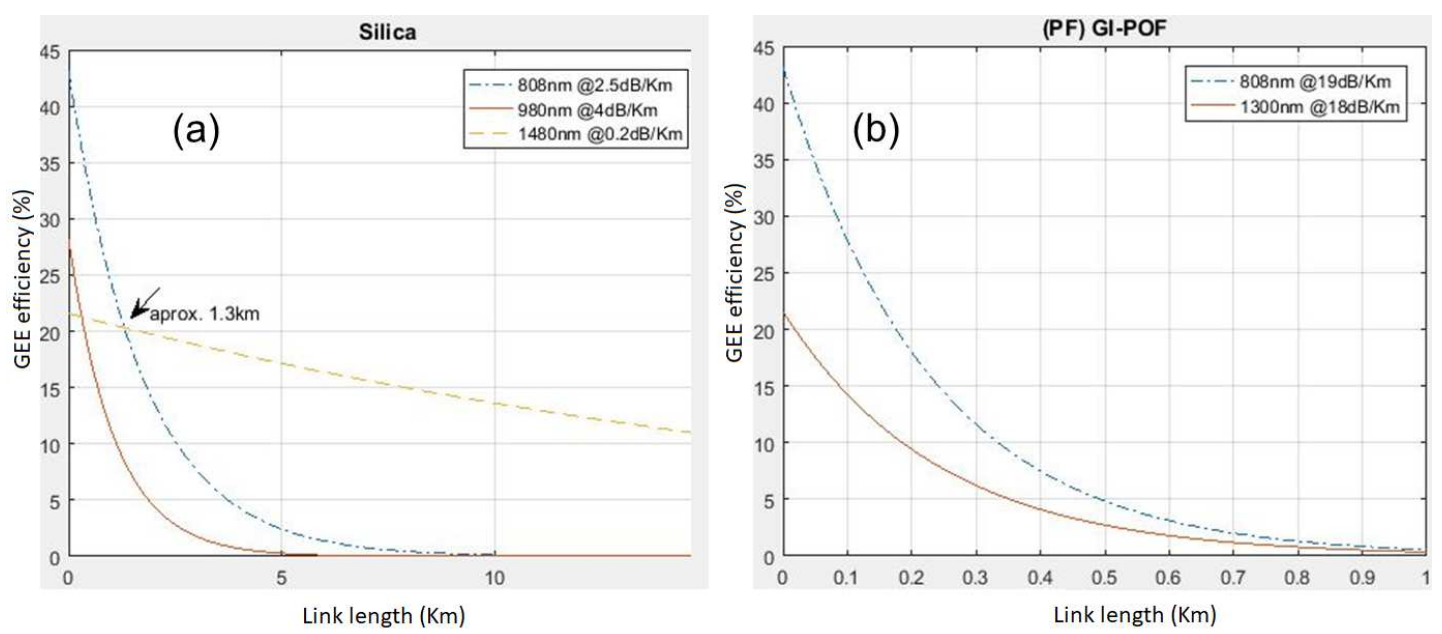

Fig. 2. Global Energy Efficiency of a PoF system with $N 1=1$, versus link length: (a) Silica fiber, (b) PF GI-POF.

GEE versus link length at different HPL wavelengths and corresponding PV converters efficiencies for silica (see Fig 2.a) and perfluorinated graded-index polymer optical fiber (PF GI-POF) are shown. It is assumed no coupling loss in any of those configurations and that optical powers are low enough to avoid fiber fuse. It can be seen that for silica fiber link lengths shorter than $1.3 \mathrm{Km}$, PV converter efficiency dominates and $808 \mathrm{~nm}$ is a good choice. This is always the case with POFs, see Fig. 2.b [3]. The 5G scenarios in autonomous driving and home networks require link lengths below $1 \mathrm{Km}$ so $808 \mathrm{~nm}$ is a good choice for those fibers. In the case of $1 \mathrm{~mm}$ SI-POF also visible light lasers can be considered [POF2018].

\subsection{Experimental set-up and results}

We have developed specific prototypes providing optical remote powering of hundreds of $\mathrm{mW}$ for different types of GOFs [5, 8] and POFs [9]. The different prototypes (see Fig. 3) include one or two high power laser diodes (HPLD) with maximum power of $2 \mathrm{~W} @ 3.3 \mathrm{~A}$, a central wavelength of $805 \mathrm{~nm}$ and with FC connectors. At the remote site there is a GaAs PV converter with an efficiency N3 of around $40 \%$. 

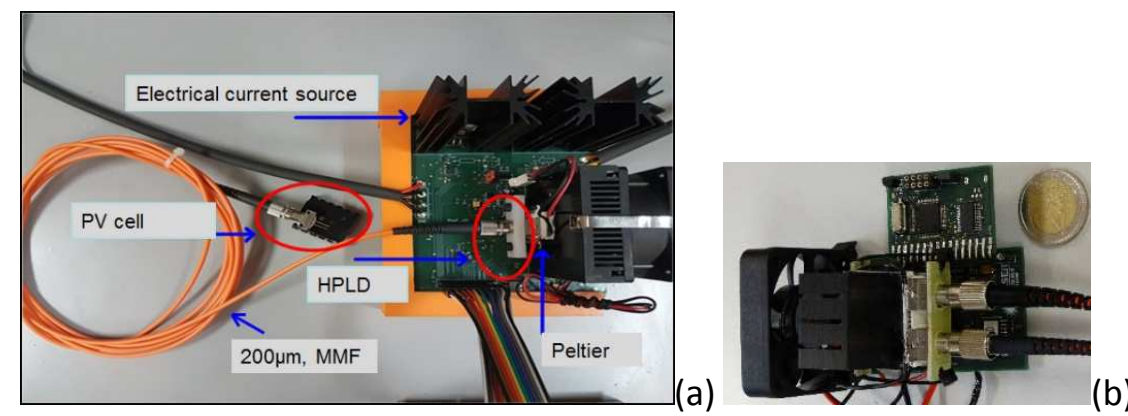

Figure 3: Photographs of prototype I (a) and II (b) of the developed PoF systems, with multimode optical fibers.

As transmission media, multimode fibers of different lengths and core diameter sizes were tested, with step index (SI) and graded index (GI) profiles. The highest transmission power density was achieved with $200 \mu \mathrm{m}$ core diameter SI-GOF. This fiber, with a 0.22 numerical aperture, also provides the maximum coupling efficiency from the HPLD output. Table 1 summarizes the set of experimental trials carried out for the different fiber types and the electrical power $\left(P_{E l e c}^{P V}\right)$ delivered to the load.

Table 1: Measurements of electrical power fed at the load for different optical fibers. 1.5W HPL at 805nm

\begin{tabular}{|c|c|c|}
\hline Fibers types [Core diameter] & Fiber link lengths[m] & Electrical power, $P_{\text {elec }}^{\text {PV }}[\mathrm{mW}]$ \\
\hline GI-GOF $[62.5 \mu \mathrm{m}]$ OM1 & 1 (ó 3 revisar-PW2017) & 240 \\
\hline SI-GOF $[200 \mu \mathrm{m}]$ & 1 & 409 \\
\hline SI-GOF $[200 \mu \mathrm{m}]$ & 300 & 340 \\
\hline SI-POF $[1 \mathrm{~mm}]$ & 2.1 & 197 \\
\hline GI-POF $[120 \mu \mathrm{m}]$ & 50 & 50 \\
\hline GI-POF $[120 \mu \mathrm{m}]$ & 30 (ó 25-PW2017) & $100(75 \mathrm{~mW})$ \\
\hline
\end{tabular}

The PF GI-POF with $120 \mu \mathrm{m}$ core diameter system started melting at around $528 \mathrm{~mW}$ of optical power injected into the fiber, in agreement with [6]. The PoF prototypes fed various remote sensor nodes in hazardous environments [5] and IoT applications [8], or an optical switch in a pyrometer [10].

\subsection{Heating effects}

Depending on the application, the energy radiated in the form of heat by the PoF system can affect the surrounding environment. Fig. 4 shows the thermal heating of a coated plastic optical fiber and two GOFs. The good coupling efficiency of $200 \mu \mathrm{m}$ core fiber implies less thermal heating, as expected.
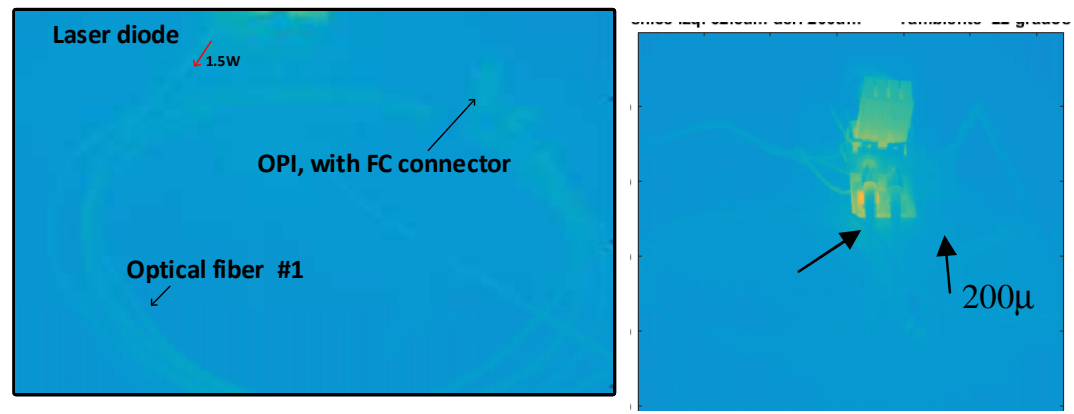

Figure 4: Thermal radiation profile with an ambient temperature of $22^{\circ} \mathrm{C}$ and $1.5 \mathrm{~W}$ at each HPLD: (a) $10 \mathrm{~m} \mathrm{SI-}$ POF fiber, $1 \mathrm{~mm}(b)$ close to HPLD with GOF fiber with two core diameters- right $200 \mu \mathrm{m}$ and left $62.5 \mu \mathrm{m}$.

\section{SINGLE MODE OPTICAL FIBER SCENARIOS}

\subsection{Specific characteristics}

The maximum amount of power that can be delivered to the remote site depends on the transmission loss, the optical fiber damage threshold and also the non-linear effects. The threshold power of both Stimulating Brioullin Scattering (SBS) and Stimulating Raman Scattering (SRS) increase with the effective area and decrease with the link length [11]. If longer lengths are considered, GEE is dominated by N2 and higher efficiencies are achieved at $1480 \mathrm{~nm}$ (see Fig. 2.a). HPL linewidth needs to be selected to avoid SBS. The effective area is smaller in 
SMFs in comparison with MMFs, but higher powers can be achieved if multiple cores of MCFs are used for optical power delivering. Future 5G mobile fronthauling based on MCF is proposed, including its integration with PoF technology and Software Define Networks [12].

\subsection{Experimental results}

Shared and dedicated PoF scenarios have been proposed as part of the infrastructure to be used for future 5G cellular networks. Either bundles of SMFs or multiple single mode cores of MCFs are used. In the shared scenario each core/fiber delivers HPL optical power and RoF data to the remote site. Different tests on 20m 4core MCFs are performed to explore the influence of HPL signals at $1480 \mathrm{~nm}$ and $2 \mathrm{~nm}$ linewidth, on a $2.6 \mathrm{Gbps}$ bit-rate data traffic signal from a SFP transceiver operating at $1550 \mathrm{~nm}$. Negligible BER penalty is measured between switching the HPL off and on, for maximum power levels of $200 \mathrm{~mW}$ [13]. The same results are obtained if a dedicated PoF scenario where data and power signals are delivered at different cores. For longer distance some tests are performed on bundles of SMFs, at $25 \mathrm{Km}$ link lengths and SRS appears at certain power levels making the signal to noise ratio to degrade, showing small BER penalties up to power levels of $100 \mathrm{~mW}$ that increases for higher HPL power levels.

\section{CONCLUSIONS}

The potential of PoF technology in some 5G verticals is analyzed using different types of optical fibers including POFs, SMFs, MMFs and MCF silica optical fibers. More than $300 \mathrm{~mW}$ of electrical power are optically delivered to remote nodes 300 meters away using multimode fibers. In the case of PF GI-POF, 50mW are delivered 50m away. Some experiments on SMFs and MCFs show that the impact on quality signal in share core/fiber scenarios are negligible for optical power levels below $100 \mathrm{~mW}$.

\section{ACKNOWLEDGEMENTS}

The authors would like to acknowledge the support of the H2020 EU-funded project BlueSPACE (grant no. 762055), by the Spanish Ministerio de Economía, Industria y Competitividad and Comunidad de Madrid under grants TEC2015-63826-C3-2-R and Y2018/EMT-4892, TEFLON-CM, FSE.

\section{REFERENCES}

[1] Cisco VNI Forecast, "Cisco Visual Networking Index: Global Mobile Data Traffic Forecast Update, 2013-2018," Cisco Public Information, (2014).

[2] D. Novak and R. Waterhouse"Emerging Disruptive Wireless Technologies - Prospects and Challenges for Integration with optical Networks," Proc. OFC/NFOEC p. OTu3E.2 (2013).

[3] C. Vázquez, D. S. Montero, P. J. Pinzón, J. D. López-Cardona, P. Contreras and A. Tapetado, "Integration of power over fiber on RoF systems in different scenarios," in Proc. SPIE 10128 Broadband Access Communication Technologies, San Francisco, CA, USA, paper 101280E, 2017.

[4] T. Umezawa et al., "Multi-Core Based 94-GHz Radio and Power over Fiber Transmission Using 100-GHz Analog Photoreceiver," in Proc. 42nd ECOC, Dusseldorf, pp. 1-3, 2016.

[5] J. D. López-Cardona, C.Vázquez, D. S. Montero and P. C. Lallana, "Remote Optical Powering Using Fiber Optics in Hazardous Environments," J. Lightwave Technol., 36(3), pp. 748-754, 2018.

[6] Y. Mizuno et al "Propagation mechanism of polymer optical fiber fuse" Scientific Reports, (2014).

[7] C. Vázquez, Fahad M. A. Al-Zubaidi, D. S. Montero1, P. C. Lallana, P. J. Pinzón, J. Mateo and J. Zubia "Characterization for simultaneous Gigabit data transmission and energy delivery in Large core Plastic Optical Fibers" in 27th Internacional Conference on Plastic Optical Fibres, 2018.

[8] Juan D. López-Cardona, David S. Montero, and C. Vázquez "Smart Remote Nodes Feed by Power over Fiber in Internet of Things Applications", IEEE Sensors Journal, in press, 2019.

[9] J. D. López-Cardona, D. S. Montero, P. J. Pinzón, and C. Vázquez, "GIPOF-based Power Delivery Systems," in 25th Internacional Conference on Plastic Optical Fibres, 2016.

[10] Carmen Vázquez, Sandra Pérez-Prieto, Juan D. López-Cardona, Alberto Tapetado, Enrique Blanco, Jorge Moreno-López, David S. Montero and Pedro C. Lallana "Fiber-optic pyrometer with optically powered switch for temperature measurements," Sensors, vol. 18, no. 22, 2018, Art. no. 483.

[11] G. Agrawal, "Nonlinear Fiber Optics", Elsevier, 2013.

[12] G. Otero, J.D. López-Cardona, R. Muñoz, C. Vázquez, D. Larrabeiti ; R. Vilalta ; J.A. Hernández ; J. M. Fàbrega, "SDN-based Multi-core Power-over-Fiber (PoF) System for 5G Fronthaul: Towards PoF Pooling," in Proceedings of 44th ECOC, Rome, Italy, 2018.

[13] J.D. López-Cardona, F. Al-Zubaidi, D.S. Montero and C. Vázquez "Experiments on Shared and Dedicated Power over Fiber Scenarios in Multi-core Fibers", EuCNC, 2019. 